\title{
NMR SHIM COIL DESIGN UTILISING A RAPID SPHERICAL HARMONIC CALCULATION METHOD
}

\author{
C. J. SNAPE-JENKINSON ${ }^{1}$, S. CROZIER ${ }^{2}$ and L. K. FORBES ${ }^{3}$
}

(Received 3 May 1999; revised 21 July 2000)

\begin{abstract}
A rapid spherical harmonic calculation method is used for the design of Nuclear Magnetic Resonance shim coils. The aim is to design each shim such that it generates a field described purely by a single spherical harmonic. By applying simulated annealing techniques, coil arrangements are produced through the optimal positioning of current-carrying circular arc conductors of rectangular cross-section. This involves minimizing the undesirable harmonics in relation to a target harmonic. The design method is flexible enough to be applied for the production of coil arrangements that generate fields consisting significantly of either zonal or tesseral harmonics. Results are presented for several coil designs which generate tesseral harmonics of degree one.
\end{abstract}

\section{Introduction}

In Nuclear Magnetic Resonance (NMR), nuclei possessing the property of 'spin' are placed in a strong static magnetic field usually produced by a superconducting magnet. Nuclei that contain an odd number of protons, neutrons or both in combination, exhibit nuclear 'spin' and a 'magnetic moment'. Some common nuclei are ${ }^{1} \mathrm{H},{ }^{2} \mathrm{H},{ }^{13} \mathrm{C},{ }^{31} \mathrm{P}$, etc.

When placed in the strong magnetic fields, the nuclear magnetic moments interact with the field and establish a number of energy levels $(2 I+1$ in fact, where $I$ is the spin quantum number). For ' $\mathrm{H}$ spectroscopy, which is the most common, $I=1 / 2$ and the two energy levels correspond to an attempt either to align or to counter-align with the applied field. As the molecules are in thermal motion, the resultant motion is gyroscopic or precessional in nature around the applied field direction (1). The

\footnotetext{
'Department of Mathematics, University of Queensland, St Lucia, Queensland 4072, Australia.

${ }^{2}$ Centre for Magnetic Resonance, University of Queensland, St Lucia, Queensland 4072, Australia.

${ }^{3}$ School of Mathematics and Physics, University of Tasmania, Hobart, Tasmania 7001, Australia.

(C) Australian Mathematical Society 2002, Serial-fee code 0334-2700/02
} 
equation governing the precessional behaviour is that of Larmor,

$$
\omega_{0}=\gamma B_{0},
$$

where $\omega_{0}$ is the angular precessional frequency, $\gamma$ the gyromagnetic ratio, which is specific to the nucleide under study, and $B_{0}$ the applied flux density. For protons $\left({ }^{1} \mathrm{H}\right)$, $\gamma=42.57 \mathrm{MHz} / \mathrm{T}$ and therefore spectroscopic investigations with resolution less than $10 \mathrm{~Hz}$ require the use of a magnet with purity across the sample of about $1.3 \times 10^{-8}$ if spectral lines at $17.6 \mathrm{Tesla}(750 \mathrm{MHz})$ are to be distinguished. This is an extreme requirement.

It is not possible to construct magnets with such precision. In addition, the introduction of the sample into the magnet perturbs the field. In the 1950's Garrett [6] and contemporaries introduced a series of electromagnets to the magnet system to attempt to reduce the impurity of the field. The electromagnets termed 'shim coils' are intended to generate spherical harmonics of the $B_{z}$ field component and thereby exploit the orthogonal properties of these functions. Theoretically, these coils should not interact when energised.

Typically, shim coils are designed by methods such as manipulating sets of equations in order to annul two or three undesirable harmonics or placing conductors at specific locations to suppress source terms describing individual harmonics [1,7-9]. Unfortunately, the resultant design of these coils was such that they interacted and were therefore impure. The consequence of this is that the 'shimming' process is long and tedious and often does not give the optimum result. In modern systems as many as 30 shim coils may be used.

The purpose of this work was to investigate the use of large scale optimization to design shims that are relatively pure and reduce interactions as far as possible. Calculating the magnetic field produced by these coils is straightforward in principle, however the important feature presented here is that the spherical harmonic representation of the magnetic field is computed directly. This by-passes the need to compute the field itself and instead yields the coefficients of a harmonic decomposition at once. The method is ideally suited to the main task of designing shims that generate only a single harmonic component. Details of the algorithm are given in Section 2 and the optimization technique for designing the required pure fields outlined in Section 3. The more difficult of these designs are the tesseral coils (those that do not have total azimuthal inclusion) and the design results of several such shim coils are presented.

\section{The spherical harmonic calculation method}

The method for calculating spherical harmonics for a given coil arrangement is crucial when designing shim coils by optimization. We improve upon techniques used in the past by utilising a new rapid calculation method which we outline here. 
Shim coils are constructed from a number of connected current-carrying circular arc conductors, fashioned from bundles of wire, which we assume to have rectangular cross-section. In NMR we are mainly concerned with the field in the direction of the magnet axis (by convention in the $z$-direction) and so may ignore any conductors lying along the $z$-direction. Thus, the spherical harmonics generated by an entire coil are given simply by the sum of contributions from each circular arc conductor forming that coil.

The field in a volume through which no current passes, and hence the field component in the $z$-direction, satisfies Laplace's equation $\nabla^{2} B_{z}=0$. Solving this for a field point $r$, measured in spherical polar coordinates $(r, \theta, \phi)$, gives the expression for the magnetic induction inside some sphere of radius $r_{0}$ centred at the origin

$$
B_{z}(r)=\sum_{n} \sum_{m} r^{n}\left[a_{n m} \cos (m \phi)+b_{n m} \sin (m \phi)\right] P_{n m}(\cos \theta) .
$$

Here, the $P_{n m}$ are orthogonal solutions (order $n$, degree $m$ ) to Legendre's associated differential equation. In this expression the terms $r^{n} a_{n m}$ and $r^{n} b_{n m}$ define respectively the size of the cosine and sine dependent spherical harmonics. Thus the coefficients $a_{n m}$ and $b_{n m}$ give the spherical harmonics of the $z$-component of the magnetic induction field within some sphere $r<r_{0}$. In practice, the sphere of radius $r_{0}$ is known as the DSV (diameter sensitive volume) and it defines the region of interest inside the magnet system where samples would be placed.

Using the orthogonal properties of (2), on the surface of a sphere with radius $r_{0}$, we obtain for $a_{n m}$ and $b_{n m}$ the formulae involving double integrals, for degree $m=0$,

$$
\begin{aligned}
& a_{n 0}=r_{0}^{-n} \frac{2 n+1}{4 \pi} \int_{\phi=0}^{2 \pi} \int_{\theta=0}^{\pi} B_{z}\left(r_{0}\right) P_{n}(\cos \theta) \sin \theta d \theta d \phi, \\
& b_{n 0}=0,
\end{aligned}
$$

and for degree $m=1,2,3, \ldots$,

$$
\begin{aligned}
& a_{n m}=r_{0}^{-n} \frac{(2 n+1)}{2 \pi} \frac{(n-m) !}{(n+m) !} \int_{\phi=0}^{2 \pi} \int_{\theta=0}^{\pi} B_{z}\left(r_{0}\right) P_{n m}(\cos \theta) \sin \theta \cos (m \phi) d \theta d \phi, \\
& b_{n m}=r_{0}^{-n} \frac{(2 n+1)}{2 \pi} \frac{(n-m) !}{(n+m) !} \int_{\phi=0}^{2 \pi} \int_{\theta=0}^{\pi} B_{z}\left(r_{0}\right) P_{n m}(\cos \theta) \sin \theta \sin (m \phi) d \theta d \phi .
\end{aligned}
$$

These equations provide the means to calculate the spherical harmonic components of $B_{z}$ within the sphere of interest. Here the vector $r_{0}$ is defined in spherical polar coordinates by $\left(r_{0}, \theta, \phi\right)$.

From Maxwell's equations, the magnetic induction $B$ is given by

$$
B(r)=\operatorname{curl} A(r),
$$


where the vector potential $A$, in a medium with permeability $\mu$, may be calculated by the generalised Biot-Savart law

$$
A(r)=\frac{\mu}{4 \pi} \iiint_{V} \frac{J(\rho)}{|r-\rho|} d V^{\prime}
$$

Our interest lies in determining the spherical harmonics produced by a current-carrying circular arc of rectangular cross-section, such as that shown in Figure 1, where source points $\rho$ are measured in cylindrical polar coordinates $(\rho, \psi, z)$. From the constant current density $J_{0}$ directed around the arc, the current density vector in (8) may be replaced by

$$
J(\rho)=J_{0} e_{\psi} .
$$

Translating the unit direction vector $\boldsymbol{e}_{\psi}$ to the spherical polar coordinate system $(r, \theta, \phi)$ used to describe field points, the vector potential given by (8) becomes

$$
A(\boldsymbol{r})=A_{r} \boldsymbol{e}_{r}+A_{\theta} \boldsymbol{e}_{\theta}+A_{\phi} \boldsymbol{e}_{\phi},
$$

with components

$$
\begin{aligned}
& A_{r}=\frac{\mu J_{0}}{4 \pi} \int_{\psi=\psi_{1}}^{\psi_{2}} \int_{\rho=r_{1}}^{r_{2}} \int_{z=z_{1}}^{z_{2}} \frac{\sin \theta \sin (\phi-\psi)}{|r-\rho|} \rho d z d \rho d \psi, \\
& A_{\theta}=\frac{\mu J_{0}}{4 \pi} \int_{\psi=\psi_{1}}^{\psi_{2}} \int_{\rho=r_{1}}^{r_{2}} \int_{z=z_{1}}^{z_{2}} \frac{\cos \theta \sin (\phi-\psi)}{|r-\rho|} \rho d z d \rho d \psi, \\
& A_{\phi}=\frac{\mu J_{0}}{4 \pi} \int_{\psi=\psi_{1}}^{\psi_{2}} \int_{\rho=r_{1}}^{r_{2}} \int_{z=z_{1}}^{z_{2}} \frac{\cos (\phi-\psi)}{|\boldsymbol{r}-\rho|} \rho d z d \rho d \psi .
\end{aligned}
$$

The distance between a field and source point is measured by

$$
\begin{aligned}
|r-\rho| & =\sqrt{\alpha^{2}+\beta^{2}+\gamma^{2}} \\
& =\sqrt{r^{2}+\rho^{2}+z^{2}-2 r z \cos \theta-2 r \rho \sin \theta \cos (\phi-\psi)},
\end{aligned}
$$

where it is convenient to define

$$
\begin{aligned}
& \alpha=z-r \cos \theta, \\
& \beta=r \sin \theta \sin (\phi-\psi), \\
& \gamma=\rho-r \sin \theta \cos (\phi-\psi) .
\end{aligned}
$$

The singularity at $\boldsymbol{r}=\boldsymbol{\rho}$ does not present a problem in this case, as the radius of the DSV, on which field points lie, is somewhat smaller in magnitude than the radii of the source conductors.

An expression for $B_{z}$ can be obtained by applying (7) which, translated to the Cartesian coordinate system, yields, for the field component in the $z$-direction,

$$
B_{z}(r)=\frac{\cos \theta}{r \sin \theta}\left(\frac{\partial}{\partial \theta}\left(\sin \theta A_{\phi}\right)-\frac{\partial A_{\theta}}{\partial \phi}\right)-\frac{1}{r}\left(\frac{\partial A_{r}}{\partial \phi}-\sin \theta \frac{\partial}{\partial r}\left(r A_{\phi}\right)\right) .
$$




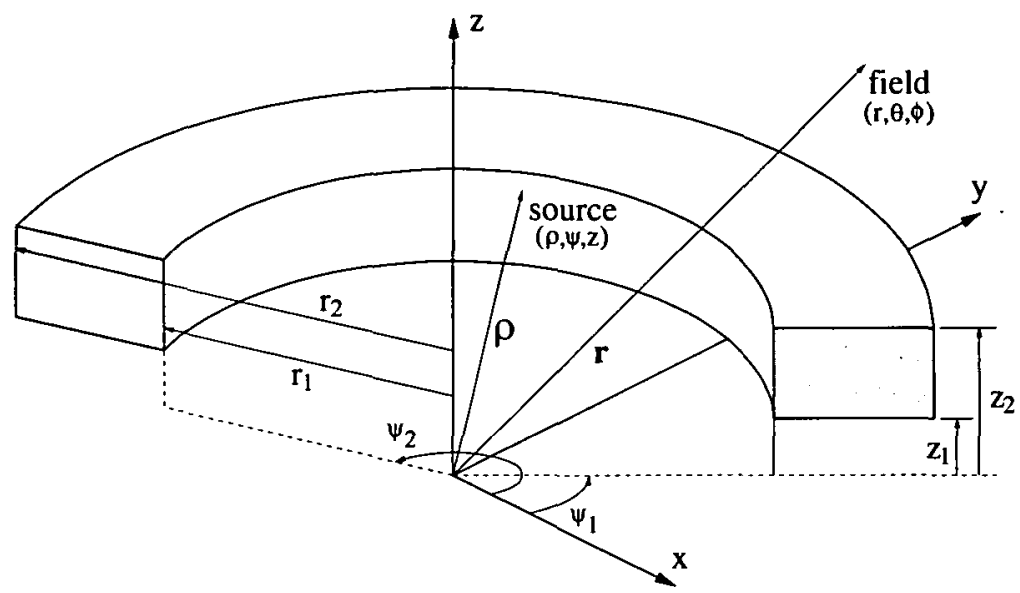

FIGURE 1. A typical circular arc with rectangular cross-section. Indicated are a source point $\rho$, measured in cylindrical polar coordinates, and field point $r$, measured in spherical polar coordinates.

This completes the equations required to describe the spherical harmonics produced by the circular arc conductor indicated in Figure 1.

Combining (3)-(6), (11)-(13) and (16) gives, for the coefficients $a_{n m}$ and $b_{n m}$ the expressions, for $m=0$,

$$
\begin{aligned}
& a_{n 0}=r_{0}^{-(n+1)} \frac{(2 n+1)}{4 \pi} \frac{\mu J_{0}}{4 \pi}\left(C_{n 0}^{(1)}-C_{n 0}^{(2)}+C_{n 0}^{(3)}\right), \\
& b_{n 0}=0,
\end{aligned}
$$

and for $m=1,2,3, \ldots$,

$$
\begin{aligned}
& a_{n m}=r_{0}^{-(n+1)} \frac{(2 n+1)}{2 \pi} \frac{(n-m) !}{(n+m) !} \frac{\mu J_{0}}{4 \pi}\left(C_{n m}^{(1)}-C_{n m}^{(2)}+C_{n m}^{(3)}\right), \\
& b_{n m}=r_{0}^{-(n+1)} \frac{(2 n+1)}{2 \pi} \frac{(n-m) !}{(n+m) !} \frac{\mu J_{0}}{4 \pi}\left(D_{n m}^{(1)}-D_{n m}^{(2)}+D_{n m}^{(3)}\right),
\end{aligned}
$$

where

$$
\begin{aligned}
C_{n m}^{(1)}= & \int_{\phi=0}^{2 \pi} \int_{\theta=0}^{\pi} \frac{\partial}{\partial \theta}\left[\sin \theta \int_{\psi=\psi_{1}}^{\psi_{2}} \cos (\phi-\psi) F\left(r_{0}, \theta, \phi ; \psi\right) d \psi\right] \\
& \times \cos \theta P_{n m}(\cos \theta) \cos (m \phi) d \theta d \phi, \\
C_{n m}^{(2)}= & \int_{\phi=0}^{2 \pi} \int_{\theta=0}^{\pi} \frac{\partial}{\partial \phi}\left[\int_{\psi=\psi_{1}}^{\psi_{2}} \sin (\phi-\psi) F\left(r_{0}, \theta, \phi ; \psi\right) d \psi\right] \\
& \times P_{n m}(\cos \theta) \cos (m \phi) d \theta d \phi, \\
C_{n m}^{(3)}= & \int_{\phi=0}^{2 \pi} \int_{\theta=0}^{\pi} \frac{\partial}{\partial r}\left[r \int_{\psi=\psi_{1}}^{\psi_{2}} \cos (\phi-\psi) F(r, \theta, \phi ; \psi) d \psi\right]_{r=r_{0}} \\
& \times \sin ^{2} \theta P_{n m}(\cos \theta) \cos (m \phi) d \theta d \phi .
\end{aligned}
$$


By replacing $\cos (m \phi)$ with $\sin (m \phi)$ in (21)-(23), the expressions $D_{n m}$ in (20) are obtained. Also defined here is the function

$$
F(r, \theta, \phi ; \psi)=\int_{\rho=r_{1}}^{r_{2}} \int_{z=z_{1}}^{z_{2}} \frac{\rho d z d \rho}{\sqrt{\alpha^{2}+\beta^{2}+\gamma^{2}}} .
$$

It is possible to evaluate the integrals in $\rho, \psi$ and $z$ explicitly, and details are shown in [10]. The two remaining integrals in $\phi$ and $\theta$ are evaluated numerically. GaussLegendre quadrature is used for the numerical integration, with thirty points in each integration parameter sufficient to maintain a high level of accuracy. In this instance, Gauss-Legendre quadrature proves more accurate than the periodic trapezoidal rule, since the integrands involve logarithms and arctangents which erode the accuracy of the trapezoidal rule.

As has been demonstrated previously in [10], the method shown here for calculating the spherical harmonics is both significantly faster and more accurate than the traditional alternative. This is because the field component $B_{z}$ is never computed explicitly; rather, the coefficients $a_{n m}$ and $b_{n m}$ of the harmonic expansion are obtained directly. This makes our method ideal for incorporation into an optimization procedure based on designing shims that give a pure field with respect to a particular harmonic.

\section{The design procedure}

The simulated annealing optimization technique is used for design, with the potential to find the global minimum of an error function. This occurs by allowing the optimization procedure to accept an occasional increase in the error function and hence the search path may escape from local minima. As the search proceeds, the number of accepted positive error excursions is reduced as described by some annealing schedule, with the intention that the system descends into a frozen state at the global minimum. The use of simulated annealing has been successfully demonstrated for a number of MR design applications (see [2-5]).

For the design problem presented here, a pattern of circular arc conductors is allowed to move randomly within a defined length (radial displacement is fixed) and the spherical harmonics generated by each arrangement calculated. Having developed a method for quick and accurate calculation, it is convenient to use harmonic values directly within the optimization procedure. For a given coil arrangement constructed from a number of arc conductors, we attempt to minimize all undesirable harmonics in relation to the particular harmonic that we wish the arrangement to produce, with all measurements taken on the surface of a sphere of radius $r_{0}$ centred at the origin of the design. This problem is described by the error function

$$
E=\frac{\sum\left(k_{i j} a_{i j}^{2}+l_{i j} b_{i j}^{2}\right)}{\left|a_{n m}^{3}\right|}
$$


where $a_{n m}$ is the amplitude of the desired cosine dependent harmonic, $a_{i j}$ and $b_{i j}$ are all other harmonics (that is, $a_{i j} \neq a_{n m}$ ) and $k_{i j}, l_{i j}$ are weighting factors for individual harmonics. In order to generate the sine dependent harmonic of corresponding order $n$ and degree $m$, the design is rotated by an appropriate angle about the $z$-axis. By taking the cube in the denominator, greater emphasis is placed on the amplitude of the target harmonic. This helps avoid poor designs that generate small fields by overlapping conductors with opposing current directions, which can often give small error function values. Constraints are easily enforced, by assigning the error function a large value when a particular arrangement is deemed outside of an acceptable range, and hence avoiding the computationally intensive need to calculate the harmonics.

Shim coils typically take the form of a number of conducting loop or saddle coils, with symmetry through the planes passing through the origin in each Cartesian coordinate. The direction of current flow is either symmetric or antisymmetric through the $z=0$ plane, depending upon the order and degree of the targeted spherical harmonic.

In order to calculate an optimal shim design, the required number of conducting arcs are defined with the proper symmetry. The choice of the number of loop or saddle coils is generally left to the designer, although including more components generally produces improved results. However, there are some restrictions placed on the components forming a coil depending upon the order and degree of the target harmonic. For example, zonal harmonics (degree $m=0$ ) are produced by an array of loops, while generating cosine dependent tesseral harmonics of degree $m=1$ requires saddle arrangements with current symmetry through the $x=0$ plane. As a general rule, more components are required and there is an increased difficulty in producing a successful design, mainly due to the larger number of optimization parameters and the decreasing amplitude of harmonics, as the order and degree increase. Roméo and Hoult [9] present a general overview of the type of designs required to generate spherical harmonics of various order and degree.

From (21)-(24) we can obtain a useful expression relating the azimuthal inclusion of an arc conductor to the degree of the generated spherical harmonics. For degree $m>0$, replace $\phi$ with $\phi+\psi$ in the expressions (21)-(23). Considering the periodicity of the integral for $\phi$, the $\psi$ integral can be performed exactly to give

$$
\begin{aligned}
C_{n m}^{(1)}= & \frac{1}{m} \int_{\phi=0}^{2 \pi} \int_{\theta=0}^{\pi} \frac{\partial}{\partial \theta}\left[\sin \theta \cos \phi F\left(r_{0}, \theta, \phi ; 0\right)\right] \\
& \times\left\{\sin \left[m\left(\phi+\psi_{2}\right)\right]-\sin \left[m\left(\phi+\psi_{1}\right)\right]\right\} \cos \theta P_{n m}(\cos \theta) d \theta d \phi, \\
C_{n m}^{(2)}= & \frac{1}{m} \int_{\phi=0}^{2 \pi} \int_{\theta=0}^{\pi} \frac{\partial}{\partial \phi}\left[\sin \phi F\left(r_{0}, \theta, \phi ; 0\right)\right] \\
& \times\left\{\sin \left[m\left(\phi+\psi_{2}\right)\right]-\sin \left[m\left(\phi+\psi_{1}\right)\right]\right\} P_{n m}(\cos \theta) d \theta d \phi,
\end{aligned}
$$




$$
\begin{aligned}
C_{n m}^{(3)}= & \frac{1}{m} \int_{\phi=0}^{2 \pi} \int_{\theta=0}^{\pi} \frac{\partial}{\partial r}[r \cos \phi F(r, \theta, \phi ; 0)]_{r=r_{0}} \\
& \times\left\{\sin \left[m\left(\phi+\psi_{2}\right)\right]-\sin \left[m\left(\phi+\psi_{1}\right)\right]\right\} \sin ^{2} \theta P_{n m}(\cos \theta) d \theta d \phi .
\end{aligned}
$$

The common expression $\sin \left[m\left(\phi+\psi_{2}\right)\right]-\sin \left[m\left(\phi+\psi_{1}\right)\right]$ vanishes when

$$
\psi_{2}-\psi_{1}=2 \pi k / m \text {, }
$$

where $m$ is the degree of a spherical harmonic and $k$ an integer. By (29) it is possible to choose the azimuthal inclusion $\psi_{2}-\psi_{1}$ such that spherical harmonics of certain degrees are not generated.

Once a layout has been defined, it becomes necessary to adjust its components in order to best generate the spherical harmonic of interest. Our method is a two-stage process. The initial optimization stage allows the position of conductors forming each loop or saddle arrangement as well as the current within each arrangement to vary, while maintaining the proper current and position symmetry. Every conductor in the coil has the same rectangular cross-section, so the result determines a ratio which describes the required number of turns of wire forming each loop or saddle arrangement.

The design is analysed, considering any overlapping or unnecessary components, and the layout finalised. A second optimization stage further refines the conductor positions, while the number of wire turns (defining the cross-section of each conducting arc) remains fixed. It is often useful to restart the optimization during either of the two design stages in order to help avoid local minima.

\section{Results}

To demonstrate the method presented in this work, several designs for producing cosine dependent tesseral spherical harmonics of degree one have been optimized and analysed. Figure 2 illustrates the typical form of shim coils which generate such harmonics. These shims produce fields consisting only of cosine dependent harmonics with odd degree. Current symmetry or antisymmetry through the $z=0$ plane result in harmonics of only odd or even order respectively. Harmonics do not exist when the degree is greater than the order (a property of associated Legendre functions) and in general the amplitude of the harmonics decreases as the order and degree increases. Therefore, excluding the undesirable harmonics of degree one, the harmonics of degree three are responsible for the greatest contamination in the generated field. By (29) it can be seen that an azimuthal inclusion of 120 degrees for the arc conductors forming a shim eliminates all harmonics of degrees $3,6,9$, etc. By considering these properties, only the necessary harmonic calculations need be performed within the optimization 


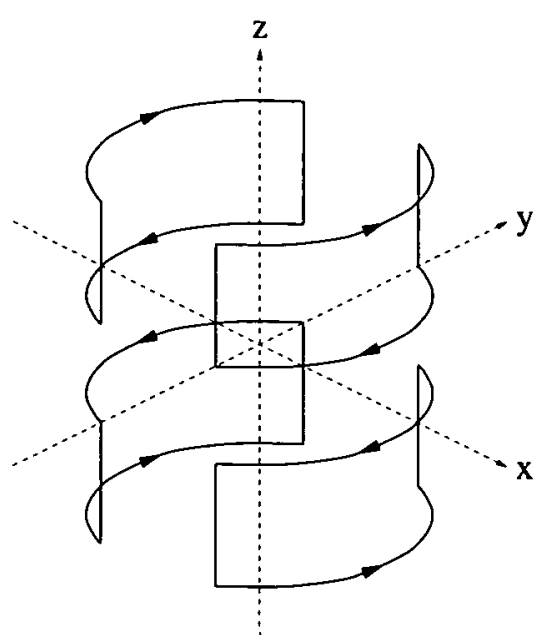

(a)

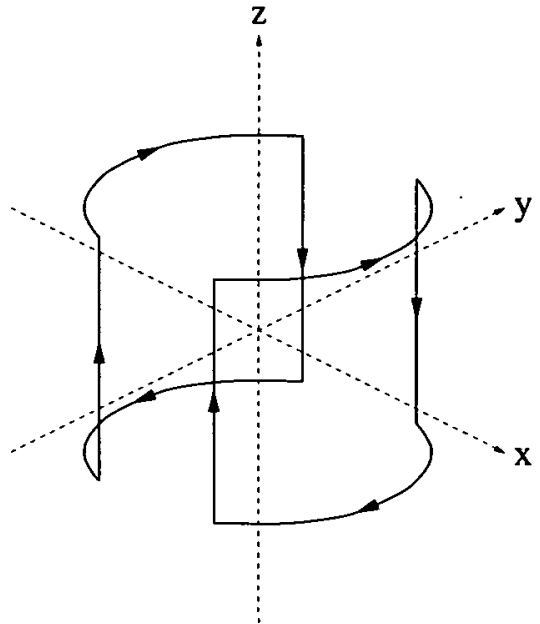

(b)

FIGURE 2. Shim coil designs constructed from symmetric arrangements of saddle coils which generate fields described primarily by tesseral spherical harmonics of degree one. The arrows indicate the current direction required to generate harmonics of (a) odd order and (b) even order.

process. For the shim designs considered here, all contributing harmonics up to order and degree seven are indicated in the tabulated results.

Once complete, a design may be scaled to any appropriate size. This allows measurements to be taken in reference to a general unit length. For the first design stage, the mean radius of the arc conductors had length one and other measurements were multiples of this. The conductors had a square cross-section with sides $1 / 1000$ and all spherical harmonic measurements were taken on a sphere with radius $1 / 3$. The $z$-positions of the conductors were restricted to a range within a displacement from the $z=0$ plane of 2.5 times the conductor radius. In each symmetric set of saddle coils the current was allowed to vary between \pm 1 . The number of iterations used in each application of the optimization routine varied from one design to the next, generally depending upon the number of optimization variables required for each design. Figure 3 shows a typical error function descent to an optimal position.

Shim designs which generate spherical harmonics of order one or two and degree one have been optimized. These shims are commonly called by the names $\mathrm{X}$ for the order one, degree one coil and ZX for the order two, degree one coil (see [3]). Two shim designs for each target harmonic, based on the layouts indicated in Figure 2, were considered. Harmonic results are shown in Table 1. For the $X$ coil, the 8 arc design shown in Figure 2 (a) has arc conductors with current magnitude 1.0 at $z$ displacements .38 and 2.5. The 16 arc design, which introduces a second set of saddle coils, has conductors with current .5 at displacements .22 and 1.38 , and current 1.0 at 


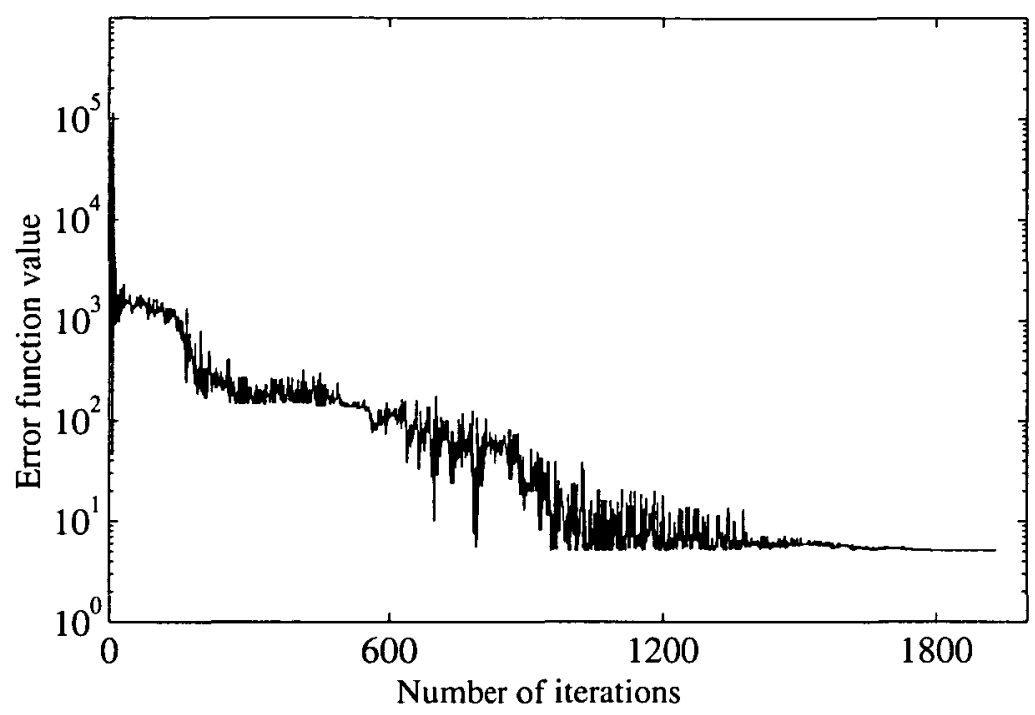

FIGURE 3. A typical error function path for shim coil design optimization. The example shown here is for the first optimization of the four arc ZX shim (order two, degree one) described in the text, with the results shown in Table 1.

displacements .82 and 2.29. The 4 arc ZX coil shown in Figure 2 (b) has conductors with current 1.0 at displacement .68 . The 8 arc ZX coil, constructed from two pairs of the saddle coils, has conductors with current .2 at displacement .46 and current 1.0 at displacement 1.16. Optimization took several hours on a multi-user Sun Ultra 5.

These results can be used as a starting point for more specific designs. Current ratios are used to define the number of wire turns forming each set of saddle coils and the conductor positions scaled to a size more useful to describe a final design. The number of turns of wire determines a fixed cross-section for the arc conductors, with details such as the specific wire layering to be considered. The $z$-displacements are refined by further optimization.

To demonstrate the process we consider the 8 arc X shim and 4 arc ZX shim. For both shim coils the number of wire turns forming each arc conductor is the same for every conductor. To allow some variation in possible cross-section, each conductor is formed from two turns of wire in one or two radial layers. The inner radius of each conductor is $5 \mathrm{~cm}$ and the wire diameter $2 \mathrm{~mm}$. Thus a two turn layering gives a rectangular cross-section with sides $4 \mathrm{~mm}$ in the $r$ direction and $2 \mathrm{~mm}$ in the $z$ direction. These values are reversed when the wires are layered side by side in the $z$-direction for one radial layer. All harmonic measurements are taken on a sphere with radius $1 / 3$ the inner radius of the conductors. Optimization occurs only in the $z$-displacements (current need no longer be considered) and refined values for the 
TABLE 1. The spherical harmonics generated by the shim coil designs described in the text, expressed as a percentage of the target cosine dependent harmonic. The number of circular arc conductors forming each coil is indicated in brackets.

\begin{tabular}{|c|c|c|c|c|}
\hline \multirow{2}{*}{$\begin{array}{c}\text { Harmonic } \\
\text { order, degree }\end{array}$} & \multicolumn{2}{|c|}{ Percentage of 1,1 } & \multicolumn{2}{c|}{ Percentage of 2,1 } \\
\cline { 2 - 5 } & X shim (8) & X shim (16) & ZX shim (4) & ZX shim (8) \\
\hline 1,1 & 100.0 & 100.0 & - & - \\
2,1 & - & - & 100.0 & 100.0 \\
3,1 & .0270 & .0499 & - & - \\
4,1 & - & - & .0049 & .0600 \\
5,1 & .3082 & .0009 & - & - \\
5,5 & .0002 & .0002 & - & - \\
6,1 & - & - & .2999 & .0000 \\
6,5 & - & - & .0000 & .0001 \\
7,1 & .0354 & .0001 & - & - \\
7,5 & .0000 & .0000 & - & - \\
7,7 & .0000 & .0000 & - & - \\
\hline
\end{tabular}

$z$-displacements obtained. With the scaling from the original displacements in mind, the $z$-displacements for the $X$ coil arcs shown in Figure 2 (a) are $2.0 \mathrm{~cm}$ and $12.8 \mathrm{~cm}$ for the one layer conductors, and $2.0 \mathrm{~cm}$ and $12.7 \mathrm{~cm}$ for the two layer conductors. For the ZX coil shown in Figure 2(b), both the one and two layer designs have arcs with $z$-displacement $3.5 \mathrm{~cm}$. The spherical harmonics generated by these designs are shown in Table 2.

\section{Conclusion}

We have demonstrated the use of simulated annealing optimization in conjunction with our rapid spherical harmonic calculation method for the design of NMR shim coils. These coils are designed to generate magnetic fields described by harmonics of particular order and degree with a minimum of impurity. Our method proves flexible enough to design shim coils that produce primarily spherical harmonics of any order and degree. Also, the rapid and accurate harmonic calculation method allows as many harmonics as required to be incorporated into the optimization process. This method need not be restricted to shim coil design and may be easily adapted to other problems in NMR and other areas.

Several shim coil designs have been described in this paper and the contributing spherical harmonics for the optimized designs shown in Tables 1 and 2. From Table 1 it can be seen that the contaminant harmonics are generated in amounts less than $1 \%$ of the target harmonics. This figure is less than $0.1 \%$ when the more complex designs involving an increased number of conducting arcs were considered. Designs have 
TABLE 2. The spherical harmonics generated by the wire layered shim coil designs described in the text, expressed as a percentage of the target harmonic. The number of wire layers is indicated in brackets.

\begin{tabular}{|c|c|c|c|c|}
\hline \multirow{2}{*}{$\begin{array}{c}\text { Harmonic } \\
\text { order, degree }\end{array}$} & \multicolumn{2}{|c|}{ Percentage of 1,1 } & \multicolumn{2}{c|}{ Percentage of 2,1 } \\
\cline { 2 - 5 } & X shim (1) & X shim (2) & ZX shim (1) & ZX shim (2) \\
\hline 1,1 & 100.0 & 100.0 & - & - \\
2,1 & - & - & 100.0 & 100.0 \\
3,1 & .0281 & .0299 & - & - \\
4,1 & - & - & .0041 & .1860 \\
5,1 & .2825 & .2648 & - & - \\
5,5 & .0002 & .0002 & - & - \\
6,1 & - & - & .2757 & .2558 \\
7,1 & .0311 & .0282 & - & - \\
\hline
\end{tabular}

been scaled to a size useful for real life NMR applications. Several wire winding patterns have been compared and the results demonstrate the need for consideration of such details as specific wire layering. Furthermore, we note the purity of harmonics for these designs will suffer in practice, especially for designs with few wire windings, due to physical limitations in construction.

\section{References}

[1] E. S. Bobrov and W. F. B. Punchard, "A general method of design of axial and radial shim coils for NMR and MRI magnets", IEEE Trans. Magn. 24 (1988) 533-536.

[2] S. Crozier, S. Dodd and D. M. Doddrell, "A novel design methodology for Nth order, shielded longitudinal coils for NMR", Meas. Sci. Technol. 7 (1996) 36-41.

[3] S. Crozier and D. M. Doddrell, "Gradient-coil design by simulated annealing", J. Magn. Reson. Ser. A 103 (1993) 354-357.

[4] S. Crozier, L. K. Forbes, W. U. Roffmann, K. Luescher and D. M. Doddrell, "A methodology for current density calculations in high-frequency RF resonators", Concepts Magn. Reson. 9 (1997) 195-210.

[5] L. K. Forbes, S. Crozier and D. M. Doddrell, "Calculating current densities and fields produced by shielded MRI probes", SIAM J. Appl. Maths 57 (1997) 401-245.

[6] M. W. Garrett, "Axially symmetric systems for generating and measuring magnetic fields, Part I", J. Appl. Phys. 22 (1951) 1091-1 107.

[7] M. J. E. Golay, Nuclear magnetic resonance apparatus, U.S. Patent 3569823 (1968).

[8] P. Konzbul and K. Švéda, "Shim coils for NMR and MRI solenoid magnets", Meas. Sci. Technol. 6 (1995) 1116-1123.

[9] F. Roméo and D. I. Hoult, "Magnet field profiling: Analysis and correcting coil design", Magn. Reson. Med. 1 (1984) 44-65.

[10] C. J. Snape-Jenkinson, L. K. Forbes and S. Crozier, "Direct algorithm for the rapid calculation of magnetic field spherical harmonics", IEEE Trans. Magn. 35 (1999) 4159-4165. 